\title{
Modeling the Structure and Evolution of Discussion Cascades
}

\author{
Vicenç Gómez \\ Donders Institute for Brain \\ Cognition and Behaviour \\ Radboud University Nijmegen \\ v.gomez@science.ru.nl
}

\author{
Hilbert J. Kappen \\ Donders Institute for Brain \\ Cognition and Behaviour \\ Radboud University Nijmegen \\ bertk@science.ru.nl
}

\author{
Andreas Kaltenbrunner \\ Information, Technology and \\ Society Research Group \\ Barcelona Media \\ kaltenbrunner@gmail.com
}

\begin{abstract}
We analyze the structure and evolution of discussion cascades in four popular websites: Slashdot, Barrapunto, Meneame and Wikipedia. Despite the big heterogeneities between these sites, a preferential attachment (PA) model with bias to the root can capture the temporal evolution of the observed trees and many of their statistical properties, namely, probability distributions of the branching factors (degrees), subtree sizes and certain correlations. The parameters of the model are learned efficiently using a novel maximum likelihood estimation scheme for PA and provide a figurative interpretation about the communication habits and the resulting discussion cascades on the four different websites.
\end{abstract}

\section{Categories and Subject Descriptors}

J.4 [Computer Applications]: Social and Behavioral Sciences-Sociology; G.2.2 [Mathematics of Computing]: Graph Theory-Network problems, Trees

\section{General Terms}

measurement, algorithms, human factors

\section{Keywords}

discussion cascades, threads, conversations, preferential attachment, maximum likelihood, Slashdot, Wikipedia

\section{INTRODUCTION}

Human communication patterns on the Internet are characterized by transient responses to social events. Examples of such phenomena are the discussion threads generated in news aggregators, the propagation of massively circulated Internet chain letters, or the synthesis of articles in collaborative web-based spaces such as Wikipedia.

These responses can be regarded as tree-like cascades of activity generated from an underlying social network. Typically, a trigger event, or a small set of initiators, generate a

Permission to make digital or hard copies of all or part of this work for personal or classroom use is granted without fee provided that copies are not made or distributed for profit or commercial advantage and that copies bear this notice and the full citation on the first page. To copy otherwise, to republish, to post on servers or to redistribute to lists, requires prior specific permission and/or a fee.

HT'11, June 6-9, 2011, Eindhoven, The Netherlands.

Copyright 2011 ACM 978-1-4503-0256-2/11/06 ...\$10.00. chain reaction which may catch the attention of other users who end up participating in the cascade (see Figure 1 for examples) and attract even more users. Since these cascades of comments are a direct consequence of the information flow in a social system, understanding the mechanisms and patterns which govern them plays a fundamental role in contexts like spreading of technological innovations [23], diffusion of news and opinion [11,20], social influence [1] or collective problem-solving [15].

Although information cascades have been extensively analyzed for particular domains, such as blogs [11, 20], chain letters [21], Flickr [6], Twitter [17] or page diffusion on Facebook [24], the cascades under consideration in those studies rarely involve elaborated discussions or complex interchange of opinions: generally, a small piece of information is just forwarded from an individual to its direct neighbors. To the best of our knowledge, with the exception of [16], no previous work exists on modeling the evolution and structure of long discussion-based cascades.

Here, as in [16], we consider several websites where the associated (discussion) cascades contain high level of interaction. We analyze for the first time the cascades of the popular news aggregator Slashdot, Barrapunto (a Spanish version of Slashdot) and Meneame (a Spanish Digg-clone) and the English Wikipedia. As the reader may notice, these datasets are quite heterogeneous. For instance, although posts from both Slashdot and Meneame correspond to popular news which rely on broadcasted events, Slashdot contains rich and very extensive comments, which are less frequent in Meneame. The cascades found in Wikipedia, on the other hand, represent collaborative effort towards a well defined goal: produce a free, reliable article.

In this study we address the following questions: what are the statistical patterns that determine the structure of such cascades and their evolution? Can these patterns be largely determined regardless of semantic information using a simple parametric model? Can the parameterization corresponding to a given website provide a global characterization for it?

We first provide a global analysis of the cascade behavior in the four mentioned websites. Among other results, we find that typically, the sizes of the cascades have a clear defined scale, which seems to contradict the recent results of [16]. Our analysis also highlights the importance of repetitive user participation in relation to other types of cascades and their impact on the entire social network. We also present a growth model for discussion cascades which is validated in the four datasets. Our approach is based on a simple model 


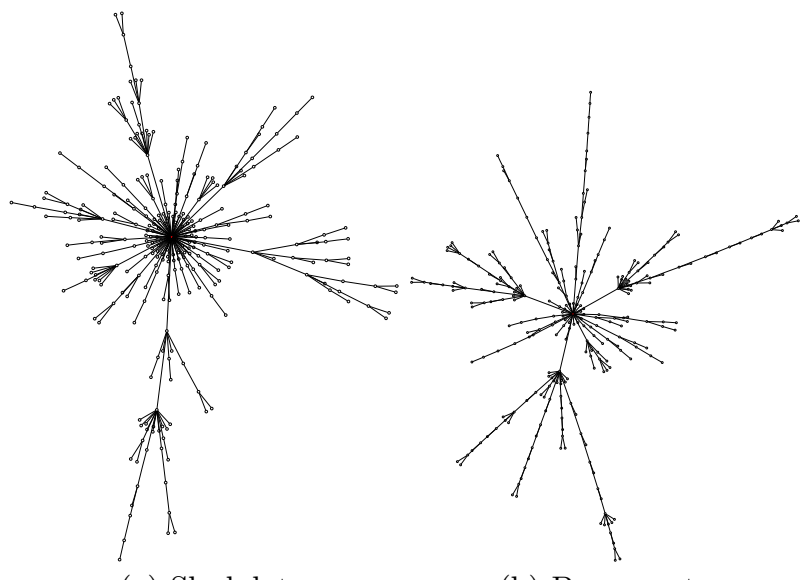

(a) Slashdot

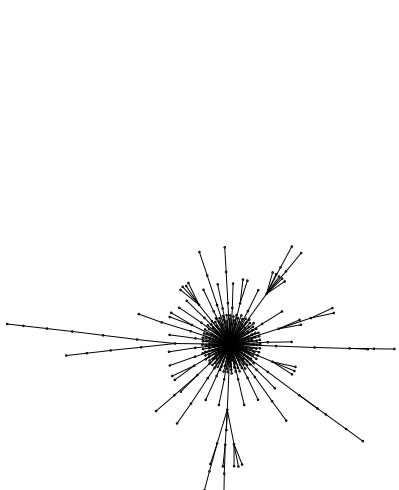

(c) Meneame

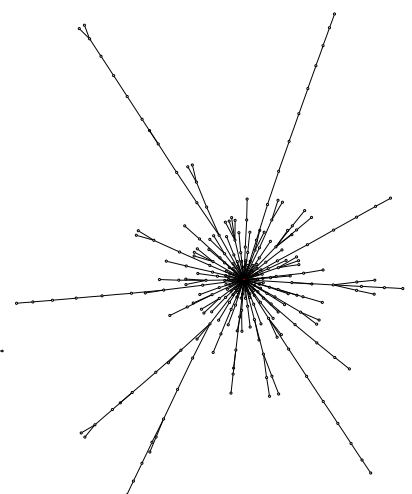

(d) Wikipedia

Figure 1: Examples of real discussion cascades.

of preferential attachment (PA) [2], where new contributions in the cascade tree are linked to existing contributions with a probability which depends on their popularity (degree).

Two key ingredients characterize our approach: First, we account for a certain bias favoring the root, or event initiator. In this way, we are able to capture the different processes governing the global (direct reactions) and the localized responses of the system. Second, we use a likelihood method particularly developed for this study which allows an efficient estimation of the model parameters which considers the entire generative model. The method is applicable not only for the data considered here but for a more general class of growing graphs. Here we are only interested on the stochastic process which generates the cascade. We do not model network dynamics or a termination criteria for the cascades. Such a model could be built on top of our current model as it is done for example in [16].

In the next Section, we explain the proposed model and how we estimate its parameters. Section 3 introduces the datasets and provides a global analysis about their main characteristics. In Section 4 we explain the main results and give an interpretation of the parameters of the model. Finally, in Section 5 we describe related work and discuss the results in Section 6. In the Appendix we explain some aspects of the likelihood approach which are important for the estimation of parameters.

\section{GROWING TREE MODELS FOR DISCUS- SION CASCADES}

We model a discussion cascade as a growing network in which nodes correspond to comments and the initial node corresponds to the post (a news article, for instance). A new node is added sequentially at discrete time-steps. Our model is based on the original PA model to which we add a bias to the first node. Since each new node adds only one new link to the existing graph, the resulting network is a tree. We also assume that the total number of nodes $N$ is known. It is convenient to represent compactly the cascade as a vector of parent nodes $\boldsymbol{\pi}$, where $\pi_{t}$ denotes the parent of the node $t+1$ added at time-step $t$.
We are interested in the probability of being node $k$ the parent $\pi_{t}$ given the past history $\boldsymbol{\pi}_{(1: t-1)}$, that is $p\left(\pi_{t}=\right.$ $\left.k \mid \boldsymbol{\pi}_{(1: t-1)}\right)$, for $t>1, k=\{1, \ldots, t\}$ and initial vector $\boldsymbol{\pi}_{1}=$ (1) ${ }^{1}$. Note that by construction, $\pi_{t} \leq t, \forall t$.

At time-step $t$, we relate the popularity of a node $k$ with its number of links (degree $d_{k, t}$ ) before node $t+1$ is added in the following way:

$$
d_{k, t}\left(\boldsymbol{\pi}_{(1: t-1)}\right)=\left\{\begin{array}{ll}
1+\sum_{m=2}^{t-1} \delta_{k \pi_{m}} & \text { for } k \in\{1, \ldots, t\} \\
0 & \text { otherwise }
\end{array},\right.
$$

where $\delta$ is the Kronecker delta function. In the following, we omit the explicit dependence on $\boldsymbol{\pi}_{(1: t-1)}$, so that $d_{k, t} \equiv$ $d_{k, t}\left(\boldsymbol{\pi}_{(1: t-1)}\right)$.

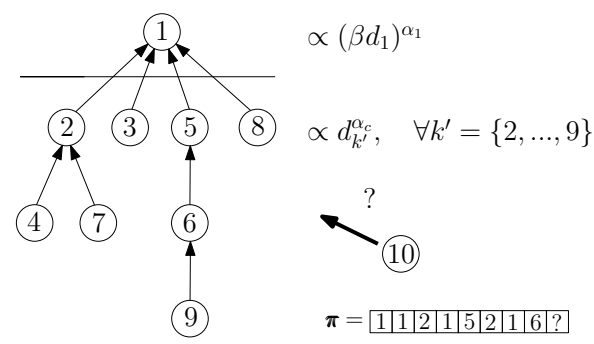

Figure 2: Small example: at time-step 9, node number 10 is added to the cascade. With probability proportional to $\left(\beta d_{1}\right)^{\alpha_{1}}$ it is added to the root node (initiator) and with probability proportional to $d_{k^{\prime}}^{\alpha_{c}}$ to one of the non-root nodes. Bottom right shows the corresponding parent vector $\pi$ (see text for definitions).

The PA model attaches new nodes to node $k$ with probability proportional to its popularity. See Figure 2 for an illustration. For completeness, we consider two models: a simple PA model without bias to the root and a model which differentiates between the root node and the rest.

\footnotetext{
${ }^{1}$ At time 0 we have $\boldsymbol{\pi}_{0}=()$ and for all trees, $p\left(\pi_{1}=1\right)=1$ and 0 otherwise, i.e. $\boldsymbol{\pi}_{1}=(1)$ always.
} 
In the general PA model, the probability for attaching the node $t+1$ to node $k$ at time-step $t>1$ is parameterized using a linear term $\beta_{k}$ and an exponent $\alpha_{k}$ for each of the nodes:

$$
p\left(\pi_{t}=k \mid \boldsymbol{\pi}_{(1: t-1)}\right)=\frac{1}{Z_{t}}\left(\beta_{k} d_{k, t}\right)^{\alpha_{k}}, \quad Z_{t}=\sum_{l=1}^{t}\left(\beta_{l} d_{l, t}\right)^{\alpha_{l}}
$$

Model without bias: If we set $\alpha_{k}=\alpha$ and $\beta_{k}=1$, for $k=\{1, \ldots, t\}$, we recover an important generalization of Barabasi's PA model, where the probability of attachment to a node goes as some general power $\alpha$ of the degree [18, 13]. For $\alpha=1$, the linear preferential attachment is recovered. In this case, nodes have power-law distributed degrees. For $\alpha<1$, or sublinear PA, the degrees are distributed according to a stretched exponential. For $\alpha>1$ there is a "condensation" phenomenon, in which a single node gets a finite fraction of all the connections in the network [18].

Model with bias: Consider the following parameterization:

$$
\begin{aligned}
& \alpha_{k}= \begin{cases}\alpha_{1} & \text { for } k=1 \\
\alpha_{c} & \text { for } k \in\{2, \ldots, t\}\end{cases} \\
& \beta_{k}=\left\{\begin{array}{ll}
\beta & \text { for } k=1 \\
1 & \text { for } k \in\{2, \ldots, t\}
\end{array} .\right.
\end{aligned}
$$

In this case, $\alpha_{1}$ and $\alpha_{c}$ are the exponents of the PA processes governing the root and the non-root nodes respectively. $\beta$ can be regarded as an additional degree of freedom weighting the root of the tree. In Section 4.4 we discuss about the interpretability of these parameters.

Note that, although we explicitly model the event which triggers the cascade as a root node, this representation does not limit the cascade to be originated from an individual event only. The root node can of course represent a group of initiators.

\subsection{Maximum likelihood parameter estimation}

Usually, PA in evolving networks is measured by calculating the rate at which groups of nodes with identical connectivity form new links during a small time interval $\Delta t[13,4]$. However, this approach is suitable only for networks with many nodes that are stationary in the sense that the number of nodes remain constant during the interval $\Delta t$. This is not a reasonable assumption in our data, which is often produced by a transient, highly nonstationary, response.

Another approach for parameter estimation relies on fitting a measured property, for instance the degree distribution, for which an analytical form can be derived in the model under consideration. For the PA model, extensive results exist with emphasis precisely on the degree distributions [3]. However, two important aspects are worth to mention here. First, analytical results usually rely on assumptions like a continuum limit or on an infinite size network, which is also not the case of our data. Second, it is important to stress here that when parameters are learned for a particular observable for which an analytical form has been derived, the model may overfit on this measure, introducing a bias in other structural properties such as subtree sizes, average depths, or other correlations.

Our approach considers the likelihood function corresponding to the entire generative process (instead of particular measures such as degree distributions or subtree sizes) introduced before. We can assign to each observation (each node arrival in each cascade) a given probability using Equation (2). The parameters for which the likelihood is maximal are the ones that best explain the data given the model assumptions (see [25] for a similar approach for another network growth model).

Formally, we observe a set $\Pi:=\left\{\boldsymbol{\pi}_{1}, \ldots \boldsymbol{\pi}_{N}\right\}$ of $N$ trees with respective sizes $\left|\boldsymbol{\pi}_{i}\right|, i \in\{1, \ldots N\}$ and we want to find the values of $\boldsymbol{\theta}:=\left(\alpha_{1}, \alpha_{c}, \beta\right)$ which best explain the data. The likelihood function can be written as:

$$
\begin{aligned}
\mathcal{L}(\boldsymbol{\Pi} \mid \boldsymbol{\theta}) & =\prod_{i=1}^{N} p\left(\boldsymbol{\pi}_{i} \mid \boldsymbol{\theta}\right) \\
& =\prod_{i=1}^{N} \prod_{t=2}^{\left|\boldsymbol{\pi}_{i}\right|} p\left(\pi_{t, i} \mid \boldsymbol{\pi}_{(1: t-1), i}, \boldsymbol{\theta}\right) \\
& =\prod_{i=1}^{N} \prod_{t=2}^{\left|\boldsymbol{\pi}_{i}\right|}\left(\beta_{x} d_{x, t, i}\right)^{\alpha_{x}}\left(\sum_{l=1}^{t}\left(\beta_{l} d_{l, t, i}\right)^{\alpha_{l}}\right)^{-1}
\end{aligned}
$$

where $\boldsymbol{\pi}_{(1: t-1), i}$ is the vector of parents in the tree $i$ after time $t-1, x:=\pi_{t, i}$ is the parent of node $t+1$ in the tree $i$, and $d_{x, t, i}:=d_{x, t}\left(\boldsymbol{\pi}_{(1: t-1), i}\right)$ denotes the degree of node $x$ as in Equation (1) in the tree $i$. Instead of maximizing (4) directly, it is more convenient to minimize the negative of the log-likelihood function:

$\log \mathcal{L}(\boldsymbol{\Pi} \mid \boldsymbol{\theta})=\sum_{i=1}^{N} \sum_{t=2}^{\left|\boldsymbol{\pi}_{i}\right|} \alpha_{x}\left(\log \beta_{x}+\log d_{x, t, i}\right)-\log Z_{t, i}\left(\boldsymbol{\pi}_{i} \mid \boldsymbol{\theta}\right)$,

where $Z_{t, i}\left(\boldsymbol{\pi}_{i} \mid \boldsymbol{\theta}\right)=\sum_{l=1}^{t}\left(\beta_{l} d_{l, t, i}\right)^{\alpha_{l}}$.

For more details about the optimization see the Appendix.

\section{DATASETS}

We have analyzed the discussion cascades of four websites. In the following paragraphs we give a more detailed description of the datasets and the corresponding websites. Global descriptive statistics can be found in Table 1.

Slashdot (SL) : Slashdot ${ }^{2}$ is a popular technology-news website created in 1997 that publishes frequently short news posts and allows its readers to comment on them. Slashdot has a community based moderation system that awards a score to every comment and upholds the quality of discussions. The comments can be nested which allows us to extract the tree structure of the discussion. A single news post triggers typically about 200 comments (most of them in a few hours) during the approx. 2 weeks he is open for discussion. Our dataset contains the entire amount of discussions generated at Slashdot during a year (from August 2005 to August 2006). See [8] for more details about this dataset.

Barrapunto (BP) : Barrapunto $^{3}$ is a Spanish version of Slashdot created in 1999. It runs the same open source software as Slashdot, making the visual and functional appearance of the two sites very similar. They differ in the language they use and the content of the news stories displayed, which normally does not overlap. The volume of activity on Barrapunto is significantly lower. A news story on Barrapunto triggers on average around 50 comments. Our

\footnotetext{
2 http://slashdot.org/

${ }^{3}$ http://barrapunto.com/
} 
Table 1: Dataset statistics for Slashdot (SL), Barrapunto (BP), Meneame (MN) and Wikipedia (WK).

\begin{tabular}{|c|c|c|c|c|c|c|}
\hline dataset & \#cascades & \#nodes (comments) & max. nodes & max. users & total users & repeated user \\
\hline$\overline{\mathrm{SL}}$ & 9,820 & $2,028,518$ & 1,567 & 1,031 & 93,638 & $>1 \quad 99 \%$ \\
\hline $\mathrm{BP}$ & 7,485 & 357,951 & 841 & 180 & 6,864 & $>1 \quad 85 \%$ \\
\hline $\mathrm{MN}$ & 58,613 & $2,220,714$ & 2,718 & 1,021 & 53,877 & $\begin{array}{ll}>1 & 34 \% \\
>5 & 70 \%\end{array}$ \\
\hline WK & 871,485 & $9,421,976$ & 32,664 & 5,969 & 350,958 & $\begin{array}{ll}>1 & 34 \% \\
>5 & 96 \%\end{array}$ \\
\hline
\end{tabular}

dataset contains the activity on Barrapunto during three years (from January 2005 to December 2008).

Meneame (MN) : Meneame ${ }^{4}$ is the most successful Spanish news aggregator. The website is based on the idea of promoting user-submitted links to news (stories) according to user votes. It was launched in December of 2005 as a Spanish equivalent to Digg. The entry page of Meneame consists of a sequence of stories recently promoted to the front page, as well as a link to pages containing the most popular, and newly submitted stories. Registered users can, among other things: (a) publish links to relevant news which are retained in a queue until they collect a sufficient number of votes to be promoted to the front page of Meneame, (b) comment on links sent by other users (or themselves), (c) vote (menear) comments and links published by other users. Contrary to both BP and SL, Meneame lacks an interface for nested comments. Comments are displayed as a list so that the tree structure is hidden However, the tag \#n can be used to indicate a reply to the $n$-th comment in the comment list and to extract the tree structures we analyze in this study. To focus on the most representative cascades, we filter out stories that were not promoted, that is marked as discarded, abuse, etc. Our dataset contains the promoted stories and corresponding comments during the interval between Dec. 2005 and July 2009.

Wikipedia (WK) : The English Wikipedia ${ }^{5}$ is the largest language version of Wikipedia. Every article in Wikipedia has its corresponding article talk page where users can discuss on improving the article. For our analysis we used a dump of the English Wikipedia of March 2010 which contained data of about 3.2 million articles, out of which about 870,000 articles had a corresponding discussion page with at least one comment. In total these article discussion pages contained about 9.4 million signed comments. Note that the comments are never deleted, so this number reflects the totality of comments ever made about the articles in the dump. The oldest comments date back to as early as 2001. Comments who are considered a reply to a previous comment are indented, which allows to extract the tree structure of the discussions. Note that Wikipedia discussion pages contain, in addition to comments, structural elements such as subpages, headlines, etc. which help to organize large discussions. We eliminate all this elements and just concentrate our analysis on the remaining pure discussion trees. More details about the dataset and the corresponding data preparation and cleaning process can be found in [19]. For our experiments we selected a random subset of 50,000 articles from the entire dataset. Results did not vary significantly when using different random subsets of the data.

\footnotetext{
${ }^{4}$ http://www.meneame.net/

${ }^{5}$ http://en.wikipedia.org
}

\subsection{Global analysis}

In this section we give a brief overview about some general characteristics of the four datasets. Several indicators are shown in Table 1 . As columns 4 and 5 show, the biggest observed discussions can be composed of hundreds of comments and propagate across hundreds of users. We find the biggest discussion in Wikipedia, involving 5,969 users and 32,664 comments. In Barrapunto, however, the biggest conversation comprised 180 users and 841 comments.

It is interesting to consider this quantity relative to the size of the underlying social network (compare columns 5 and 6 , where we indicate the total number of users during the crawled period). We see a remarkable fact: the percentage of users affected by the largest cascade is very small. In particular, it varies from a $1.1 \%$ for Slashdot and $2.6 \%$ in Barrapunto, the dataset which we saw that presented the smallest cascade in absolute terms. Globally, these results show that even the largest cascades only affect a very small portion of the entire underlying social network.

A characteristic feature of discussion cascades is the high frequency of user participation. Evidence of this is provided in column 7 , where we show the percentage of cascades in which at least one user is involved more than once for cascades with more than two nodes (for MN and WK, we also show the percentage for cascades with more than five nodes). With the exception of Meneame, all datasets show very high values. In Slashdot, practically all posts contained at least one user who commented more than once (considering only registered users). An important consequence of this fact is that information diffusion may not be properly explained using epidemic models such as SIR (susceptible-infectedrecovered) models unlike in other scenarios like photo popularity [5] or fanning pages [24].

Figure 3 shows the distribution of the cascade sizes of the four datasets. As expected, all distributions are pos-
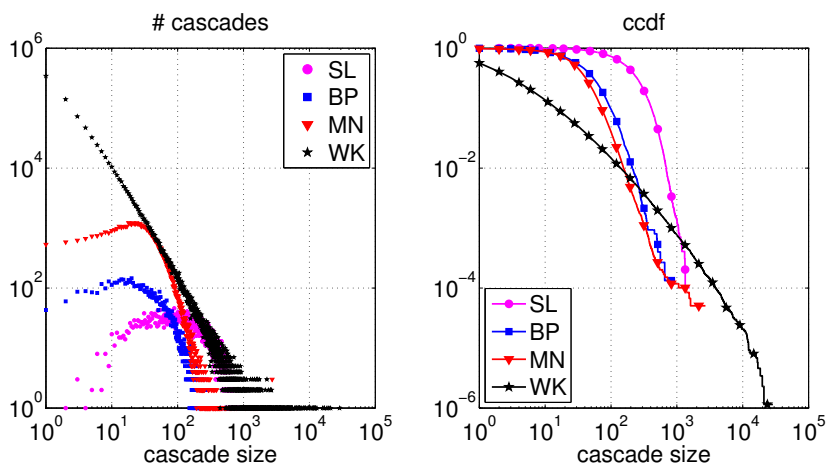

Figure 3: Cascade sizes for the different datasets 


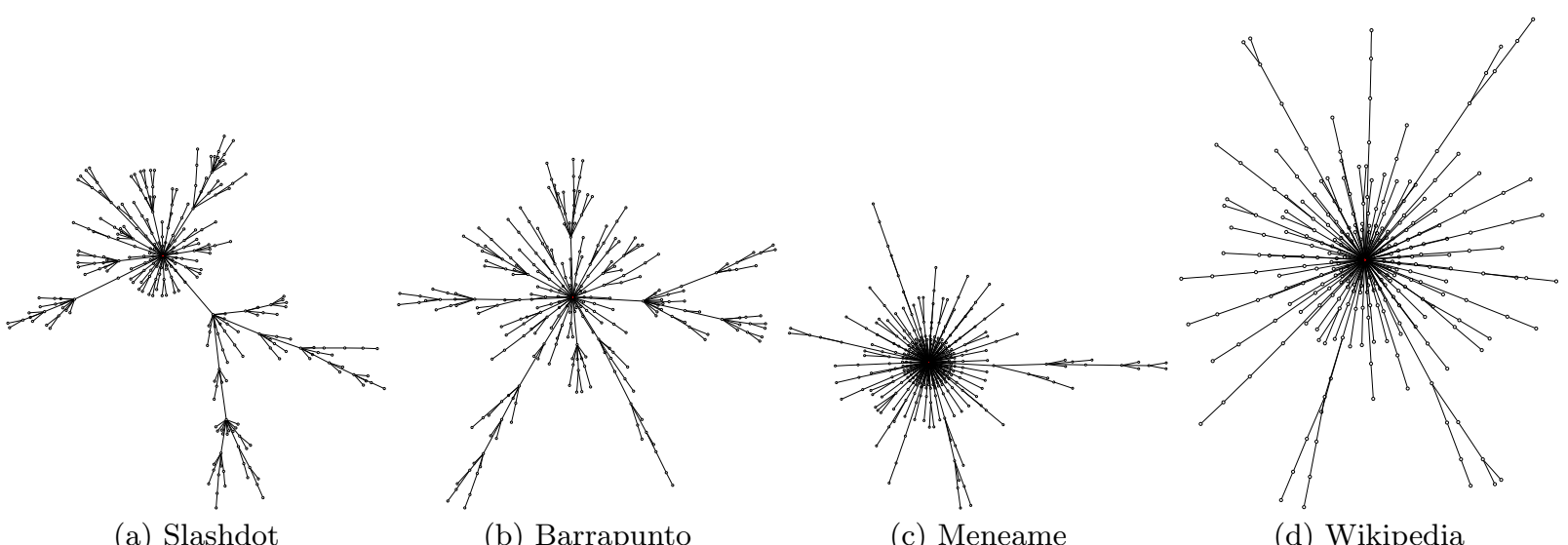

(a) Slashdot

(b) Barrapunto

(c) Meneame

(d) Wikipedia

Figure 4: Examples of synthetic discussion cascades.

itively skewed, showing a high concentration of relatively short cascades and a long tail with large cascades. However, although all distributions are heavy tailed, we clearly see a different pattern between the three news aggregators and the Wikipedia. Whereas SL, BP and MN present a distribution with a defined scale, the distribution of cascade sizes of Wikipedia is closer to a scale-free distribution, in line with the cascades found in weblogs [20] and USENET [16]. We remark that, even in the Wikipedia case, the power-law hypothesis for the tail of this distribution is not plausible via rigorous test analysis: we obtain an exponent of 2.17 at the cost of discarding $97 \%$ of the data.

We also observe a progressive deviation from websites with a well defined scale such as Slashdot, which could be described using a log-normal probability distribution, towards websites with less defined scale such as Meneame, which may show a power-law behavior for cascade sizes $>50$. Barrapunto falls in the middle and, interestingly, is more similar to Meneame than to Slashdot.

The previous considerations imply that, in general, a new post in Slashdot can hardly stay unnoticed and will propagate almost surely over several users. Conversely, most of the news in Meneame will only provoke a small reaction and reach, if they do, a small group of users. Compared with Wikipedia, we can say that Meneame is the news aggregator which has most similarities with it.

Figure 1 illustrates the different types of cascades which we found. We plot representative cascades with similar sizes selected randomly from each of the four datasets. For Slashdot we can see that the chain reaction is located mainly on the initiator event (direct reactions), but some nodes also have high degree, resulting in bursty disseminations. We could say that after a news article is posted, the collective attention is constantly drifting from the main post to some new comments which become more popular. In Barrapunto we observe similar structures, although their persistence is less noticeable. On the contrary, Meneame is characterized by having high concentration of nodes at the first level together with rare but long chains of thin threads. This represents a pattern where only a few comments receive multiple replies, but that sporadically can trigger a long dialog between a few users. We note that this phenomenon might be caused by the fact that the cascade tree and, more impor- tantly, the number of replies a comment receives are hidden in the interface of Meneame. Finally, the case of Wikipedia is very similar to Meneame, but with even longer, more frequent and finer threads of nodes with very low degree.

\section{RESULTS}

In this section we validate the proposed model by comparing the real cascades to the ones generated using the model.

\subsection{Model validation description}

We use the cascades from the four datasets to validate the proposed PA model with bias. The parameters are optimized for each dataset independently using the entire dataset and we generate the same number of synthetic cascades as the number of real cascades extracted from each dataset. An alternative validation would be to use a traintest paradigm on each dataset independently to prevent overfitting. For simplicity, and since the goal of this study is to characterize the different datasets instead of minimizing the generalization error of new threads sampled from the model, we prefer to use the entire datasets for learning. ${ }^{6}$

The size of each synthetic cascade is pre-determined drawing a pseudo-random number from the empirical distribution of cascade sizes (see Figure 3). We calculate the following quantities from the empirical data and from the synthetic cascades produced by the model:

Root node degree probability distribution: Each cascade has a root degree, which is the number of direct contributions to the root.

Total degree distribution: We consider the degree probability distribution of any node, without differentiating root versus non-root nodes.

Subtree sizes distribution: For each non-root node, we compute the probability distribution of the total number of its descendants.

Mean node depth: Each non-root node belongs to one level of the cascade. We compute the mean over all the levels of all the nodes.

\footnotetext{
${ }^{6}$ The estimated parameter values did not vary significantly using different, sufficiently large random subsets of the data, as the outcomes of a cross-validation (train-test) procedure would have produced.
} 

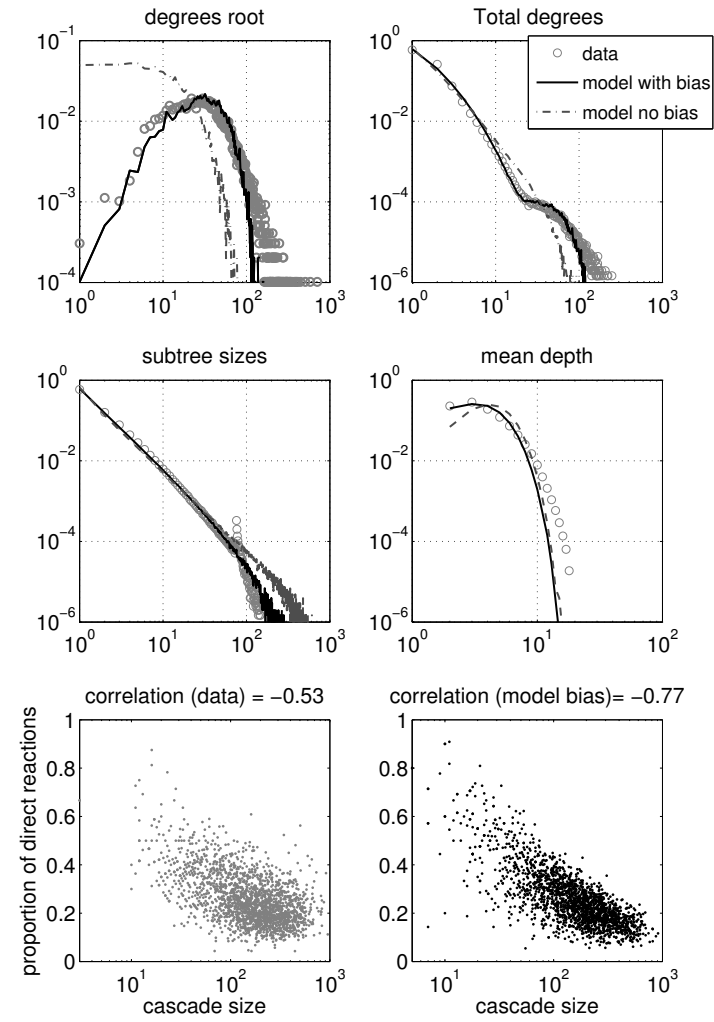

Figure 5: Model validation for Slashdot

Size - Proportion of direct reactions : We compute the relation between the size of a cascade and the proportion of direct reactions to the root and analyze if they are correlated.

\subsection{Structure of the cascades}

Figures 5, 6, 7 and 8 show plots of the previous quantities for each dataset and the outcomes of both PA models with and without bias to the root.

Overall, the model with bias is able to capture reasonably well all the measured properties, except the mean depth. In particular the degree distributions of the root nodes are very accurately reproduced, even though each dataset exhibits a different profile (see top-left plot of the figures). For this quantity, the difference between using or not a bias term is clearly manifested. A model without bias systematically produces degree distributions too skewed for the non-root nodes and with too short tails for the root nodes, and is not able to capture qualitatively the shape of the total degree distribution (see top-right plots of the figures).

A similar behavior is observed in the correlations between the log-size of the cascade and the proportion of direct reactions (bottom plots of the figures). Although the scatter plots differ substantially across datasets, the model with bias is able to reproduce them qualitatively, which is not the case for the model without bias (data not shown).

The model with bias also generates correct subtree sizes in general, with the exception of Meneame, which we postulate is caused by the particularities of the platform (see
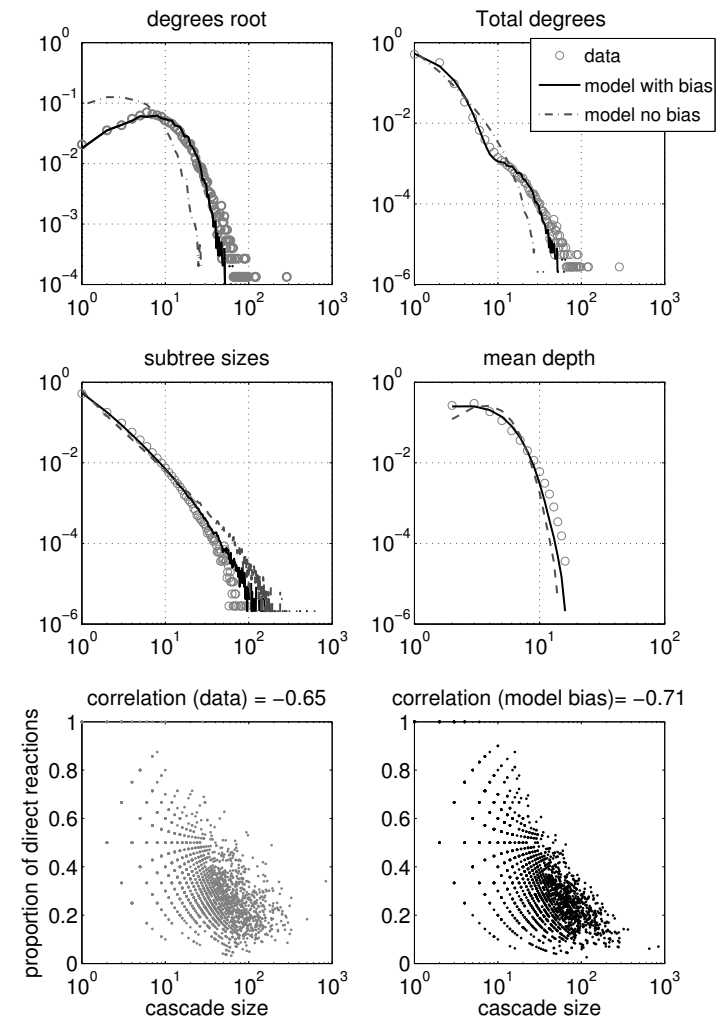

Figure 6: Model validation for Barrapunto

Section 6 for details). On the contrary, the model without bias systematically produces longer tails than the real ones.

Both models tend to produce shorter tails for the mean depth distribution in all datasets. This seems to be a current limitation of the model. Although for Slashdot and Barrapunto this deviation is not very severe, for the other two datasets we observe clear discrepancies at the tail of the distributions. Notice that in this case, the model without bias is unable even to reproduce the probability mass corresponding to the first values of the distribution. We will return to this point in Section 6 .

To conclude this section, we show in Figure 4 the synthetic counterpart of Figure 1, where we plot representative cascades with similar sizes selected randomly from each of the four synthetic datasets. We can see that the generated cascades present a strong resemblance with the real ones.

\subsection{Evolution of the cascades}

After having compared the main structural properties of the synthetic trees with the real ones, we now investigate whether the PA model with bias is also able to reproduce the growth process of the cascades. In other words, if we take intermediate snapshots of the cascades during their evolution, how close match the synthetic trees their archetypes?

To this end we record two quantities: the width (maximum over the number of nodes per level) and the mean depth of the trees every time a new node is added (at every timestep). Note that the timesteps in the model do not coincide with the actual time differences between the comments. They just reflect the sequence of the comments 

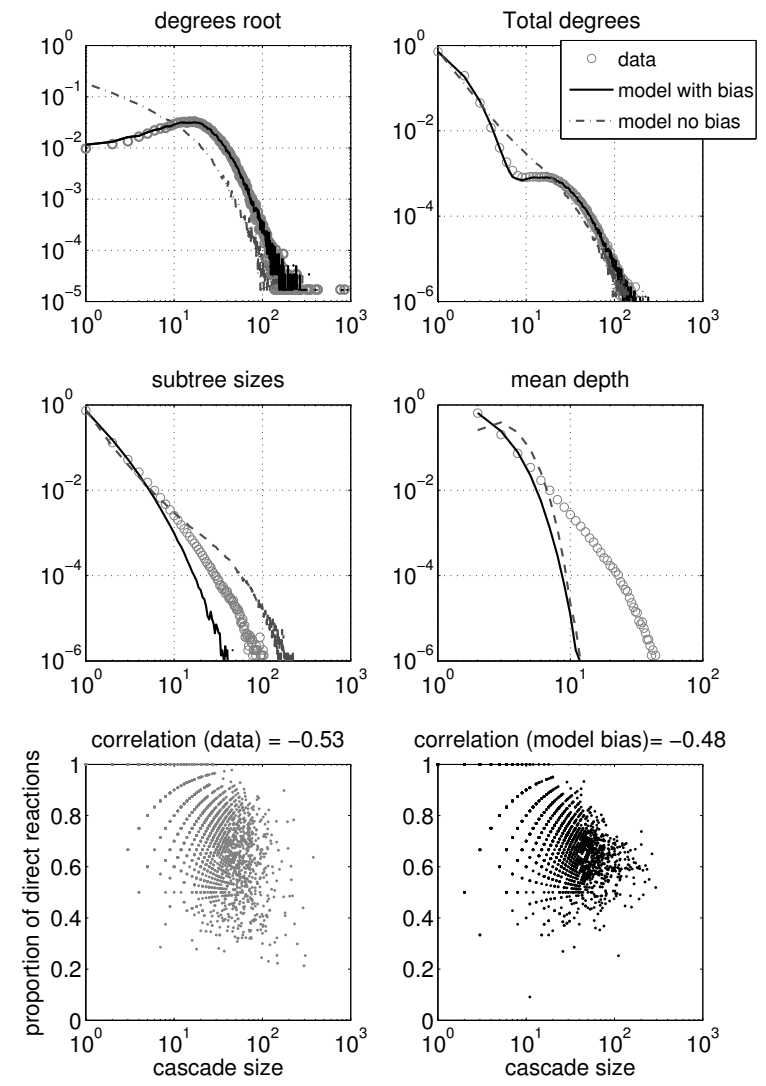

Figure 7: Model validation for Meneame

attaching to the cascade. In reality, information spreading is conditioned to the large heterogeneity present in human activity, for instance induced by circadian cycles, which results in information transmission speeds governed by subexponential distributions, i.e. log-normals or power-laws [12, $14,22]$. Capturing the growth process of the real cascades is therefore a challenging task for our model.

The average overall width and depth evolution curves are presented in Figures 9 and 10 for all datasets comparing the original cascades (continuous lines with symbols) with the biased model (dashed lines). We observe a nearly perfect coincidence between our model and the data in the evolution of the width of the discussions (Figure 9), for three of the four datasets. Only in the case of Slashdot the model underestimates the width of the tree, although it still reproduces the same curve shape if normalized by the final depth.

The picture in the case of the mean depth (Figure 10) is less favorable, but still shows a reasonable coincidence of our model with the data. In the case of Wikipedia, although the model underestimates the mean depth, it reproduces a rescaled version of it. The other datasets show a similar profile. Initially, the synthetic trees are too deep and the mean depth is overestimated. This deviation is corrected at some point and then the opposite effect takes place: when the depth of the synthetic trees saturates, the depth of the real ones still grows. The initial deviation is specially severe in Slashdot, for which remarkably the final mean depth is
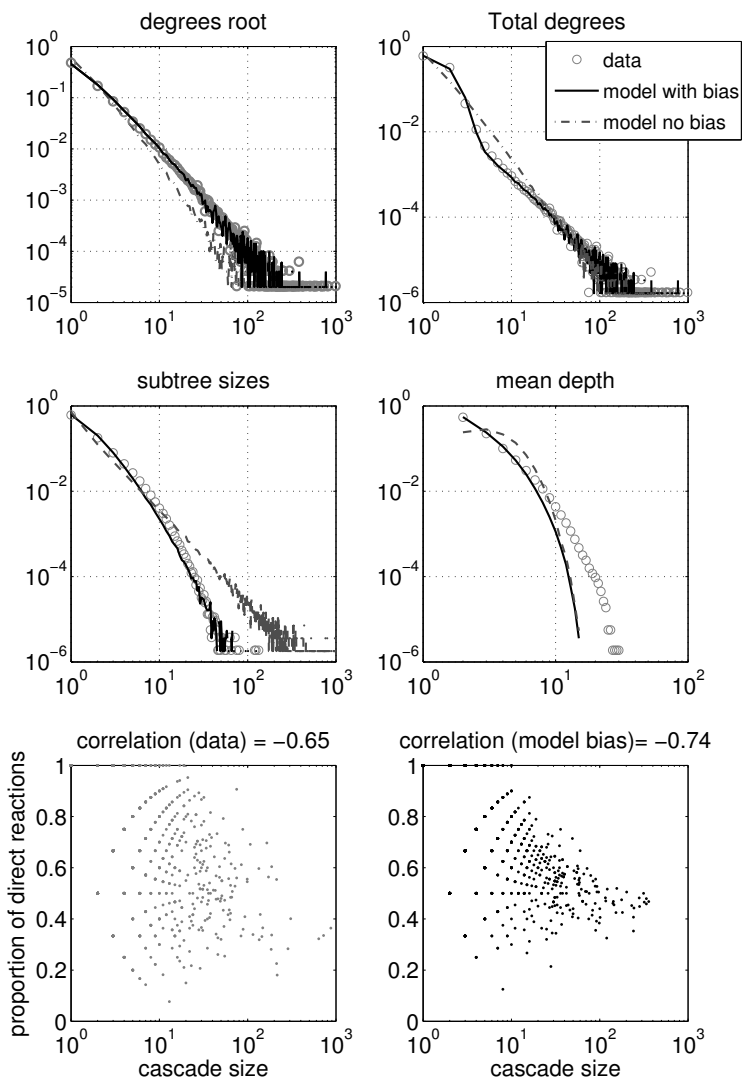

Figure 8: Model validation for Wikipedia

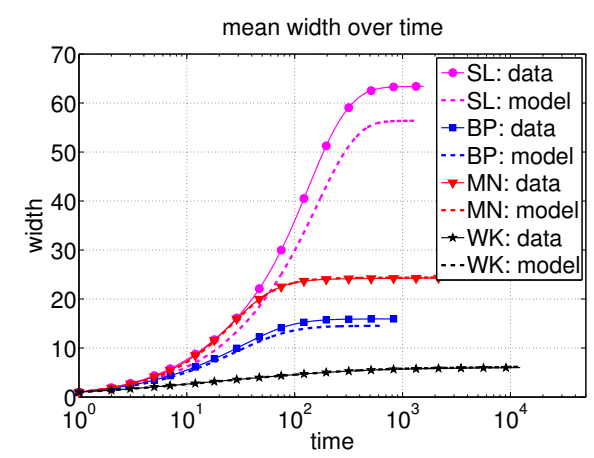

Figure 9: Evolution of the width

very close to the one of the real cascades. A possible way to overcome this problem is discussed in Section 6 .

\subsection{Interpretation of parameters}

Can we derive conclusions about the communication habits which characterize each website based on the obtained parameters which best fit each model? Figure 11(a) shows the optimal parameter values for each dataset in a three dimensional plot, where the horizontal and vertical axis correspond to $\alpha_{1}$ and $\alpha_{c}$ respectively and the size of the marker to the value $\beta$. Table 2 shows the same values numerically.

The role of the exponents $\alpha_{1}$ and $\alpha_{c}$ in the model is to quantify the degree of preferential attachment of the root 


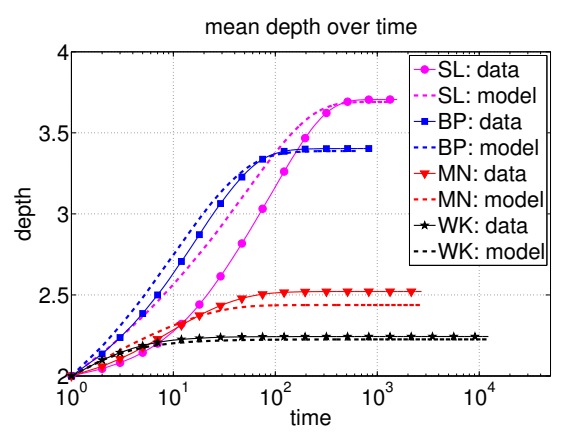

Figure 10: Evolution of the mean depth

Table 2: Optimal parameters

\begin{tabular}{|c|c|r|c|}
\hline dataset & $\alpha_{1}$ & \multicolumn{1}{|c|}{$\alpha_{c}$} & $\beta$ \\
\hline SL & 0.734 & 0.683 & 1.302 \\
\hline BP & 0.665 & -0.116 & 0.781 \\
\hline MN & 0.856 & 0.196 & 1.588 \\
\hline WK & 0.884 & -1.684 & 0.794 \\
\hline
\end{tabular}

node and the non-root nodes respectively. The higher their values, the more relevant is the popularity to determine the attractiveness to new nodes in the cascade. For instance, values very close to zero imply a random cascade where new nodes are linked to existing ones with uniform probability. We can use the established theoretical results described in Section 2 to characterize the websites under study.

The different exponents $\alpha_{1}$ are all sublinear $(<1)$, relatively high, and very similar in all datasets, indicating a strong PA in the root process of all cascades. The two lowest values for this quantity are observed for Barrapunto and Slashdot. On the other hand, Meneame and Wikipedia present a higher and almost identical value, suggesting a very similar role of the root nodes in the PA mechanism of both websites.

A clear segregation between the group of three news media websites and the Wikipedia is manifested on Figure 11(a) in the value of $\alpha_{c}$. Slashdot, has the highest value, $\alpha_{c} \approx 0.68$, even higher than $\alpha_{1}$ for Barrapunto. It is also very similar to $\alpha_{1}$ for the same dataset. This similarity may capture the special quality of the Slashdot comments. In a sense, good comments may behave like posts and may act eventually as effective initiator of information diffusion cascades.

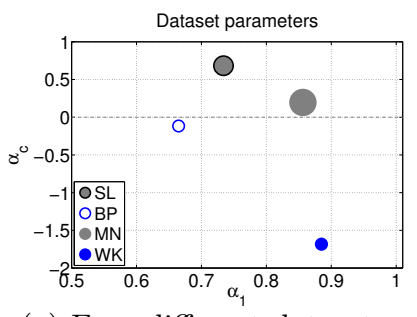

(a) Four different datasets

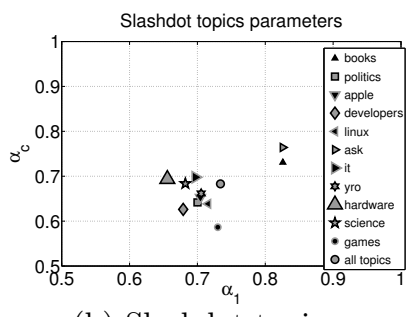

(b) Slashdot topics
Figure 11: Comparison of parameter values for (a) the different datasets and (b) the topics of Slashdot. Marker sizes encode $\beta$ differently in (a) and (b).
The smaller value of $\approx 0.2$ found for Meneame indicates that the diffusion of the news comments in this website is closer to a random process. This can be again influenced by the lack of explicit information about the popularity of a given comment. The same is true for Barrapunto although its value of $\alpha_{c} \approx-0.1$ is slightly negative, indicating a slight inverse PA process.

However, such an inverse PA process is much more prominent in the case of Wikipedia: whereas its $\alpha_{1}$ is high, indicating a strong PA in the root process in agreement with the other datasets, its $\alpha_{c}$ is negative and has the largest absolute value of all four datasets. What are the implications of this result? Once a comment on a Wikipedia article has been originated, it will derive in a collaborative reciprocal chain between a very reduced group of contributors. So once a node has received a reply it will be three (exactly: $2^{1.686}=3.2$ ) times less likely to receive another one than the replying new comment itself. In other words, nodes with degree equal to one (leaf nodes) are much more likely to be linked with a new node than nodes which higher degrees.

Finally, the parameter $\beta$ acts as a weight which expresses the trend towards the root of the cascade in relation to the subsequent nodes. It is especially important in the beginning of the cascade, when the degree of the root node is low, and determines whether initially many nodes attach to the root or rather to one of the first comments. We observe thus that Meneame shows the largest initial predominance of direct reactions, while Wikipedia gives higher probability mass to the comments, allowing thus large chains already with a small number of nodes. The values for Slashdot and Barrapunto lie in between indicating an intermediate initial preference for the root node, showing Barrapunto a higher probability for early reply-chains than Slashdot.

What would be the scenario if one tries to explain the cascades using the simple model without bias to the root? We also fit the simple PA model to the data. In that case, we would infer mistakenly that both Meneame and Wikipedia systems are in the "condensation" regime [18], since their exponents (1.360 and 1.161 respectively) are larger than one.

The next question we want to answer is how stable are these parameters within the same site, i.e. if we split for example different discussions according to their category, do we obtain similar parameters? We investigate this question for the topic categories in Slashdot (we only consider categories with more than 100 discussions) and find (see Figure 11(b)) that the majority of topics has a set of parameters which is close to the one obtained for the entire website.

This is remarkable given the heterogeneous picture that is observed if the depths and widths of the discussions are considered [9]. So it seems that, although the amount of comments which attracts the different categories may be different, the actual structure of the discussions follows a very similar pattern. However, if we consider the $\alpha$ parameters, we observe three outliers from the main cluster. The topics "books" and "ask" have much larger values, indicating a more experienced preferential attachment behavior, while the topic "games", on the other hand, has the largest difference between $\alpha_{1}$ and $\alpha_{c}$. It seems that in this topic category direct comments to the root node are more frequent ${ }^{7}$ while in the two other outliers also comments seem to be able to attract a reasonable amount of replies. It is also interesting

\footnotetext{
${ }^{7}$ This category is also the one with the shallowest trees [9].
} 
to observe the differences in the values of $\beta$, where we find the largest trend toward the root for "hardware" and the smallest for "books" articles.

Summarizing, the optimal parameters permit an interpretation of the communication habits of each social space and are relatively stable across different categories within a site. This representation also leads to different classification as a function of the parameters. The bias to the root node is crucial to separate Slashdot and Barrapunto from Wikipedia and Meneame according to $\alpha_{1}$, and Wikipedia from the three news aggregators according to $\alpha_{c}$.

\section{RELATED WORK}

Due to the increasing availability of empirical data on cascades, extensive work is appearing with focus on how information cascades are propagated in a social network.

At a statistical description level, information cascades have been analyzed in detail for particular social spaces. Twitter cascades [17] are predominantly shallow and wide (maximum depth is 11). Flickr [6] shows the remarkable phenomenon that popular photos spread slowly and not widely. This is in harmony with our findings which report that even the largest realizations reach a very small proportion of the social network.

Blog cascades have been analyzed in [20]. Interestingly, although one would expect blog cascades to share more similarities with the discussion cascades existing in Slashdot or Meneame, it is the Wikipedia dataset which shows most similar patterns to the blog cascades (see Figure 3). In [10], a model of both blogger (user) and cascades was presented which reproduces global temporal and structural aspects of the blogosphere. We note that the motivation of our work is rather different. Whereas [20, 10] aims for finding the simplest, parameter-free model able to describe both user network and cascade behavior, we look for a parameterized model from which we can describe communication habits which characterize a particular website (see Section 4.4). In contrast to the blog data, the datasets considered here contain complete information of the cascade evolution. In this sense, our data avoids selection bias which strongly influences the estimation of these processes [7]. In [7], a simple branching process (Galton-Watson process) is proposed for modeling chain-letter cascades. Although such a model may explain certain characteristics such as depths distributions (after proper correction for selection bias) it cannot capture the cascade evolution and assumes that all degree distributions are independent, so its utility for our purposes remains very limited.

During the development of this manuscript we learned of the work of Kumar et al. [16] which also presents a model for discussion trees, called T-MODEL. The same study also considers other aspects of the cascades such as the identities of each member of the conversation. Our work is focused on the cascade model, its parameter estimation and validation on the four datasets. The same or a different authorship model as the one of [16] could also be built on top of the model proposed here.

The T-MODEL is based on linear preferential attachment only, and unlike ours does not distinguish between root and subsequent nodes. However, it includes a recency term which allows it to capture qualitatively the relation between the sizes and the depths of the cascades. Preliminary experiments indicate similar ability of our model in this aspect. Additionally, the bias to the root considered here clearly permits to capture other quantities with higher accuracy, such as the degree distributions or the subtree sizes. This suggests that at least for the datasets analyzed here our model performs better. The maximum likelihood estimation scheme presented here finds the best parameters of a model given the data, and therefore allows to quantify objectively the predictive power of different models. Such a comparison between the two cascade models and possible hybrid forms is left for future research.

A further difference between the T-MODEL and our study is that it also includes a parameter for the termination of the discussion. The resulting termination probability of a discussion is independent of its actual structure and could be substituted by any other model encoding the popularity of discussions (e.g. [26]), which could also be combined with our model.

\section{DISCUSSION}

We have presented a thoughtful analysis and comparison of the structure and evolution of the different discussion cascades of three popular news media websites and the English Wikipedia. Our analysis highlights the heterogeneities between the discussion cascades, which can be conditioned from two factors, namely, the page design, or platform, and the audience. Despite this, we have given evidence that a simple model can capture most of the structural properties and the evolution profiles of the real cascades with the particularities of each dataset. Further, we have derived a rigorous maximum likelihood approach which considers the entire evolution of the cascade. The learned parameters of the model proposed here allow for a figurative description that characterizes the communication habits of a website.

For some datasets, the model tends to produce too shallow cascades. We postulate that this occurs especially in mature discussions, where interaction at the leaves only happens between a few individuals who start to reply mutually to each other and increase the mean cascade depth considerably. A possible extension which could correct for that effect is focus of current research.

\subsection{Acknowledgments}

We wish to thank David Laniado and Riccardo Tasso for providing the preprocessed Wikipedia dataset and Meneame. net for allowing to access an anonymised dump of their database. We also thank Mohammad Gheshlaghi and Alberto Llera for useful discussions.

\section{APPENDIX}

\section{Log-likelihood function}

In this appendix we describe some considerations related to the log likelihood function (5) we want to minimize. Briefly, we show that the PA model can be formulated as a probability distribution which belongs to the exponential family. Consequently, the optimization problem is convex, e.g. has the convenient property that any local minimum is global.

Without loss of generality we can assume that parameter $\beta_{k}$ is of the following form:

$$
\beta_{k}^{\alpha_{k}}:=\exp \left(\beta_{k}^{\prime}\right) .
$$


We can rewrite the PA model defined in Equation (2) as:

$$
p\left(\pi_{t}=k \mid \boldsymbol{\pi}_{(1: t-1)}\right)=\frac{1}{Z_{t}^{\prime}} \exp \left(\beta_{k}^{\prime}+\alpha_{k} \log d_{k, t}\right),
$$

where $Z_{t}^{\prime}=\sum_{l=1}^{t} \exp \left(\beta_{l}^{\prime}+\alpha_{l} \log d_{l, t}\right)$. This probability distribution is equivalent to that of the Equation (2), but expressed in terms of the exponential family. The log-likelihood function (4) can be rewritten as:

$$
\log \mathcal{L}^{\prime}(\boldsymbol{\Pi} \mid \boldsymbol{\theta})=\sum_{i=1}^{N} \sum_{t=2}^{\left|\boldsymbol{\pi}_{i}\right|} \beta_{x}^{\prime}+\alpha_{x} \log d_{x, t, i}-\log Z_{t, i}^{\prime}\left(\boldsymbol{\pi}_{i} \mid \boldsymbol{\theta}\right)
$$

where $Z_{t, i}^{\prime}\left(\boldsymbol{\pi}_{i} \mid \boldsymbol{\theta}\right)=\sum_{l=1}^{t} \exp \left(\beta_{l}^{\prime}+\alpha_{l} \log d_{l, t, i}\right)$. The Hessian of this function (matrix of second order partial derivatives) is always positive semi-definite.

The presented method can therefore be applied to any set of observations which can be expressed as a collection of parent vectors $\boldsymbol{\Pi}$ from which the degrees of each node at each time-step can be obtained. Once the minimization is performed, we can recover the original parameter $\beta_{k}$ with $\beta_{k}=\exp \left(\beta_{k}^{\prime} / \alpha_{k}\right)$. The basic PA model is the special case where $\alpha=\alpha_{1}=\alpha_{c}$.

Note that the bias to the root node can be introduced:

(A) Using two alphas $\alpha_{1}, \alpha_{c}$ but no $\beta(\beta=0)$.

(B) Using one alpha $\alpha=\alpha_{1}=\alpha_{c}$ and $\beta$.

(C) Using two alphas $\alpha=\alpha_{1}=\alpha_{c}$ and $\beta$ (the approach presented in this manuscript).

As expected, since model (C) uses more parameters than (A) and (B), the resulting likelihoods and fits are better. In particular, the impact of adding $\beta$ as a parameter is notable in the approximated measures related to the root node, for instance the root degree distributions.

Notice that the convexity does not imply uniqueness of optimal parameter values. It could happen that the same minimum is attained for a large range of parameter values. We used as an optimization procedure the Nelder-Mead simplex algorithm (implemented as fminsearch in Matlab) which is an unconstrained non-linear direct search method that does not use numerical or analytic gradients. Starting from many different random initial conditions, we did not find multiple optimal values in any of the datasets, so we can conclude that the presented values for each dataset are unique.

\section{A. REFERENCES}

[1] E. Bakshy, B. Karrer, and Lada A. Adamic. Social influence and the diffusion of user-created content. In EC '09, pages 325-334, New York, USA, 2009. ACM.

[2] A.-L. Barabási and R. Albert. Emergence of scaling in random networks. Science, 286(5439):509-512, 1999.

[3] E. Ben-Naim and P. L. Krapivsky. Stratification in the preferential attachment network. Journal of Physics A: Mathematical and Theoretical, 42(47):475001, 2009.

[4] B. F. Blasio, A. Svensson, and F. Liljeros. Preferential attachment in sexual networks. PNAS, 104(26):10762-10767, 2007.

[5] M. Cha, A. Mislove, B. Adams, and K. P. Gummadi. Characterizing social cascades in Flickr. In WOSP '08, pages 13-18, New York, USA, 2008. ACM.

[6] M. Cha, A. Mislove, and K. P. Gummadi. A measurement-driven analysis of information propagation in the Flickr social network. In $W W W^{\prime} 09$, pages 721-730, New York, USA, 2009. ACM.
[7] B. Golub and M. O. Jackson. Using selection bias to explain the observed structure of internet diffusions. PNAS, 107(24):10833-6, 2010.

[8] V. Gómez, A. Kaltenbrunner, and V. López. Statistical analysis of the social network and discussion threads in slashdot. In $W W W$ '08, pages 645-654. ACM, 2008.

[9] S. Gonzalez-Bailon, A. Kaltenbrunner, and R. E. Banchs. The structure of political discussion networks: A model for the analysis of e-deliberation. Journal of Information Technology, 25:230-243, 2010.

[10] M. Götz, J. Leskovec, M. McGlohon, and C. Faloutsos. Modeling blog dynamics. In ICWSM, 2009.

[11] D. Gruhl, R. Guha, D. Liben-Nowell, and A. Tomkins. Information diffusion through blogspace. In $W W W$ '04, pages 491-501, New York, USA, 2004. ACM.

[12] J. L. Iribarren and E. Moro. Impact of human activity patterns on the dynamics of information diffusion. Phys. Rev. Lett., 103(3):038702, Jul 2009.

[13] H. Jeong, Z. Néda, and A. L. Barabási. Measuring preferential attachment in evolving networks. Europhys. Lett., 61(4):567, 2003.

[14] A. Kaltenbrunner, V. Gómez, and V. López. Description and prediction of Slashdot activity. In $L A-W E B$ '07, Santiago de Chile, 2007. IEEE CS.

[15] M. Kearns, S. Suri, and N. Montfort. An experimental study of the coloring problem on human subject networks. Science, 313(5788):824-827, 2006.

[16] R. Kumar, M. Mahdian, and M. McGlohon. Dynamics of conversations. In $S I G K D D$ '10, pages 553-562, New York, USA, 2010. ACM.

[17] H. Kwak, Ch. Lee, H. Park, and S. Moon. What is Twitter, a social network or a news media? In $W W W$ '10, pages 591-600, New York, USA, 2010. ACM.

[18] Krapivsky P. L., Redner S., and F. Leyvraz. Connectivity of growing random networks. Phys. Rev. Lett., 85(21):4629-4632, Nov 2000.

[19] D. Laniado, R. Tasso, Y. Volkovich, and A. Kaltenbrunner. When the Wikipedians talk: Network and tree structure of Wikipedia discussion pages. In ICWSM. The AAAI Press, 2011.

[20] J. Leskovec, M. McGlohon, C. Faloutsos, N. Glance, and M. Hurst. Cascading behavior in large blog graphs: Patterns and a model. In SDM '07, 2007.

[21] D. Liben-Nowell and J. Kleinberg. Tracing information flow on a global scale using Internet chain-letter data. PNAS, 105(12):4633-4638, 2008.

[22] R. D. Malmgren, J. M. Hofman, L. A.N. Amaral, and D. J. Watts. Characterizing individual communication patterns. In SIGKDD '09, pages 607-616, New York,USA, 2009. ACM.

[23] Everett M. Rogers. Diffusion of innovations. Free Press, New York, 5th edition, 2003.

[24] E. Sun, I. Rosenn, C. Marlow, and T. M. Lento. Gesundheit! modeling contagion through facebook news feed. In ICWSM. The AAAI Press, 2009.

[25] C. Wiuf, M. Brameier, O. Hagberg, and M. P. H. Stumpf. A likelihood approach to analysis of network data. PNAS, 103(20):7566-7570, 2006.

[26] F. Wu and B.A. Huberman. Novelty and collective attention. PNAS, 104(45):17599-17601, 2007. 